e-migrinter

\title{
e-Migrinter
}

$21 \mid 2020$

Famille et migrations

\section{Famille et migrations}

Du franquisme au boom économique espagnol du XXIe siècle

Paola Garcia, Mercè Pujol Berché et Alejandro Román Antequera

\section{CpenEdition}

Journals

Édition électronique

URL : https://journals.openedition.org/e-migrinter/2426

DOI : $10.4000 /$ e-migrinter.2426

ISSN : 1961-9685

Éditeur

UMR 7301 - Migrinter

Référence électronique

Paola Garcia, Mercè Pujol Berché et Alejandro Román Antequera, «Famille et migrations », e-Migrinter

[En ligne], 21 | 2020, mis en ligne le 21 décembre 2020, consulté le 20 mai 2021. URL : http://

journals.openedition.org/e-migrinter/2426 ; DOI : https://doi.org/10.4000/e-migrinter.2426

Ce document a été généré automatiquement le 20 mai 2021.

Tous droits réservés 


\title{
Famille et migrations
}

\author{
Du franquisme au boom économique espagnol du XXIe siècle \\ Paola Garcia, Mercè Pujol Berché et Alejandro Román Antequera
}

Partant des postulats de Ravenstein $(1885 ; 1889)$, différentes théories sur l'étude des migrations se sont développées. Parmi celles-ci, la théorie néo-classique des migrations qui dans une perspective macro-économique considère que la raison essentielle des flux migratoires réside dans les différences salariales entre les pays et postule, dans une perspective micro-économique, que la migration est le résultat d'une décision individuelle prise au vu de critères économiques rationnels. Cette théorie est remise en cause dans les années 1990 par une série de travaux qui s'inscrivent dans le courant de pensée de la Nouvelle Économie des Migrations (Stark, 1984) et qui offre de nouvelles perspectives théoriques. L'acteur de référence n'est plus l'individu mais c'est la dynamique familiale qui est désormais privilégiée par les chercheurs. Dès lors, la décision d'émigrer n'est plus perçue comme le résultat d'un choix individuel mais comme le résultat d'une action collective. L'interaction entre le migrant et sa famille est alors analysée comme une stratégie permettant d'augmenter les ressources et de minimiser les risques. Ce changement de perspective analytique qui considère la migration relevant de logiques collectives (familiales et communautaires) donne lieu à de nouvelles approches, entre autres, autour de la notion de réseaux (Massey, 1990), de la perspective de genre (Sassen, 2000) et du transnational (Schiller; Basch; BlancSzanton, 1992).

2 Pour approfondir la thématique de la famille et des migrations, nous avons co-organisé un colloque international les 11 et 12 janvier 2018: «Migration: une histoire de famille». L'objectif scientifique était d'approfondir la question des migrations en considérant précisément la famille comme élément central d'analyse à partir de champs disciplinaires variés (sociologie, histoire, anthropologie, littérature, sociolinguistique, etc.) et d'aires géographiques multiples (Amérique latine, France, Espagne, Afrique). De nombreuses questions ont orienté les réflexions, à savoir : quels sont les avantages et les coûts de la migration pour l'unité familiale? Quelle intégration pour le groupe familial dans le pays de réception? La migration favorise-t-elle la 
construction de nouvelles structures familiales? Quels sont les transferts culturels que suppose ce processus, en particulier pour les jeunes générations?

3 Cela nous a conduit à travailler autour de quatre axes : a) Famille et reconfigurations familiales ; b) Famille, rapports de genre et rapports intergénérationnels ; c) Famille, politiques publiques et politiques d'intégration et d) Famille et cycles migratoires.

4 Ces quatre axes de réflexion ne sont nullement étanches. En effet, la féminisation des migrations a mis non seulement en évidence la reconfiguration des familles et les modifications et restructurations du rôle des femmes, devenues chefs de famille, mais également le changement de rôle des hommes restés dans le pays d'origine. De même, la famille élargie est amplement mise à contribution car bien souvent, c'est elle qui a la charge des enfants que les émigrés ont laissés au pays, mais c'est elle également qui accueille les épouses des émigrés (comme c'est le cas par exemple au Sénégal), ce qui aboutit à ce que ces femmes soient, la plupart du temps, soumises aux normes de la belle famille. La négociation des rôles joués par tous les membres de la famille élargie est l'une des conséquences de la constitution de familles transnationales. Ces transformations produisent de profondes mutations sociales et sociétales, les relations de pouvoir changent, tout comme les liens de parenté et le poids de l'appartenance sociale et identitaire. $\mathrm{Du}$ point de vue méthodologique, l'étude des familles transnationales suppose des recherches multi-situées et l'utilisation d'outils d'analyse complexes qui ouvrent de nouvelles pistes de recherche.

5 Quant aux politiques menées aussi bien par les pays de résidence que par les pays d'origine, elles sont souvent restrictives, soumises la plupart du temps aux lois du marché du travail et ne prennent pas vraiment en compte l'intégralité du noyau familial. C'est le cas des travailleurs saisonniers qui sont obligés de rejoindre le pays d'origine après quelques mois passés dans le pays d'accueil. De même, l'absence de permis de séjour ne permet pas aux familles de faire la demande de regroupement familial. La souffrance s'installe ainsi dans ces familles qui vivent la douleur de la séparation.

6 Les différentes recherches présentées lors des séances de travail du colloque ont contribué à éclairer ces questions et nous avons souhaité réunir quelques-unes de ces contributions pour constituer ce numéro de la revue e-Migrinter qui interroge plus spécifiquement le cas de l'Espagne, traditionnel pays d'émigration, devenu pays d'immigration dans les années 1990. Les articles de ce numéro traitent plus particulièrement de la période allant du franquisme au boom économique espagnol du début du XXIème siècle et mettent en lumière différents cas permettant d'analyser le phénomène migratoire sur le temps long et à partir de différents espaces géographiques (Espagne/Amérique latine, Espagne/Sénégal, Espagne-Galice/CatalogneFrance), laissant entrevoir les continuités et les discontinuités du phénomène. Le film qui est aussi présent dans ce numéro retrace les parcours migratoires sous l'angle de la diversité (individus, famille, amis, réseaux) et met en lumière les difficultés liées à la fois au départ et à l'intégration.

$7 \quad$ Le premier article du dossier se centre sur l'expérience migratoire espagnole au cours du franquisme. Il évoque l'expérience migratoire des Galiciens qui deviennent l'image par antonomase du migrant espagnol. Dans son article, Emma Rubio retrace l'histoire migratoire d'une famille galicienne et castillane. Elle explique comment s'est produit le démantèlement familial et l'oubli des savoirs ancestraux lors du parcours migratoire, qui amena la famille d'abord vers la Catalogne, puis vers la France. 
8 Les deux autres articles du dossier se concentrent sur l'Espagne comme pays d'accueil, en tenant compte non seulement des processus d'intégration des individus et des familles, mais également des conséquences du départ du migrant pour la société d'origine. Ces articles abordent deux cas concrets, celui du Sénégal et celui de la Colombie, le dernier pays ayant constitué un des principaux flux de migrants latinoaméricains vers l'Espagne.

Celio Sierra-Paycha analyse l'expérience migratoire des Colombiens vers l'Espagne entre 1998 et 2007 à partir des réseaux familiaux. Il montre que l'entrée par les dispersions familiales permet d'identifier et de différencier les parcours et les étapes de développement du système migratoire. Le réseau familial s'avère déterminant dans la consolidation et le déploiement massif de flux migratoires d'une région précise vers une destination particulière car il rend familier ce nouvel espace migratoire pour les candidats au départ. Il montre, grâce à son travail de terrain, que les migrants identifiés comme "aventuriers" produisent de la discontinuité dans les trajectoires familiales tandis que d'autres parcours migratoires s'avèrent plus structurés se déployant au sein de réseaux familiaux déjà constitués dans les pays de réception.

10 L'article de Marème Niang-Ndiaye explore l'incidence de la migration internationale sur les mutations sociales et sociétales au Sénégal et met en évidence les tensions qui apparaissent dans le contexte des mariages à distance. L'auteure analyse et compare les conséquences de la migration sur les femmes mariées sénégalaises restées au pays et celles parties vivre seules à Barcelone. Elle montre que le maintien des mariages à distance est soumis à une série de négociations constantes face aux contraintes socioéconomiques et socio-culturelles des espaces migratoires. La situation "maribataire ", terme par lequel on désigne le conjoint resté au Sénégal, suppose un défi complexe et incertain pour ce pays.

11 Enfin, le documentaire intitulé Mémoires en exil et réalisé en 2016, constitue une réflexion sur la mémoire (et les mémoires) des enfants espagnols exilés en France et en URSS au cours de la Guerre Civile espagnole. Le documentaire est constitué de cinq entretiens qui mettent en lumière l'expérience migratoire sous l'angle des images et de la parole et non du texte. Néanmoins l'analyse discursive et sémiologique que l'on peut en faire présente des similitudes avec des analyses du discours propres à d'autres champs scientifiques.

\section{BIBLIOGRAPHIE}

Massey, Douglas (1990) Social structure, household strategies, and the cumulative causation of migration, Population Index, vol. 56, $\mathrm{n}^{\circ}$ 1, pp. 3-26.

Ravenstein, E.G. (1885) The laws of migration, Journal of the Royal Statistical Society, vol. 48, pp. 167-227.

Ravenstein, E.G. (1889) The laws of migration (revised), Journal of the Royal Statistical Society, vol. 52, pp. 241-301.

e-Migrinter, 21 | 2020 
Sassen, Saskia (2000) Women's burden : counter-geographies of globalization and the feminization of survival, Journal of International Affairs, vol. 53, $n^{\circ} 2$, pp. 503-524.

Schiller, N.G. ; Basch, L. ; Blanc-Szanton, C. (1992) Transnationalism : a new analytic framework for understandding migration, Annals of the New York Academy of Sciences, vol. 645, n 1, pp. 1-24.

Stark, Oded (1984) Discontinuity and the theory of international migration, Kylos, vol. 37, $\mathrm{n}^{\circ} 2$, pp. 206-222.

\section{AUTEURS}

\section{PAOLA GARCIA}

Université Paris Nanterre, UR Etudes Romanes

pgarcia@parisnanterre.fr

\section{MERCÈ PUJOL BERCHÉ}

Université de Perpignan Via Domitia, CRESEM

\section{ALEJANDRO ROMÁN ANTEQUERA}

Université Paris Est Créteil, IMAGER

alejandro.roman-antequera@u-pec.fr 